\title{
Ocorrência de anticorpos e fatores de risco associados à infecção por Leptospira spp. em Cebus spp. mantidos em cativeiro no Nordeste do Brasil ${ }^{1}$
}

\author{
Débora R.A. Ferreira², Plautino de O. Laroque ${ }^{2}$, Paulo G.C. Wagner ${ }^{3}$, Severino S. dos \\ S. Higino ${ }^{4}$, Sérgio S. Azevedo ${ }^{4}$, Eneida W. Rego ${ }^{2}$ e Rinaldo A. Mota ${ }^{2 *}$
}

\begin{abstract}
Ferreira D.R.A., Laroque P.O., Wagner P.G.C.W., Higino S.S.S., Azevedo S.S., Rego E.W. \& Mota R.A. 2011. [Occurrence of antibodies and risk factors associated with Leptospira spp. infection in capitivity Cebus spp. from Northeast of Brazil.] Ocorrência de anticorpos e fatores de risco associados à infecção por Leptospira spp. em Cebus spp. mantidos em cativeiro no Nordeste do Brasil. Pesquisa Veterinária Brasileira 31(11):1019-1023. Laboratório de Doenças Infecciosas dos Animais Domésticos, Departamento de Medicina Veterinária, Universidade Federal Rural de Pernambuco, Rua Dom Manuel de Medeiros s/n, Dois Irmãos, Recife, PE 52171-900. E-mail: rinaldo.mota@hotmail.com

This study aimed to verify the occurrence of anti-Leptospira antibodies and the risk factors associated with infection in the Cebus spp. mantained in captivity in the northeast of Brazil. One hundred and thirty nine blood samples of different species of primates of both sexes and varied ages were analyzed. For the study of antibodies the Microscopic Agglutination Test (MAT) was used, and for the risk factor study a multivariate analysis was used. Antibodies anti-Leptospira, serovar Patoc were observed in 6.5\% of primates (09/139). The risk factor identified in this study was the lack of daily hygienization of drinking fountains (Odds ratio=12.095; IC 95\% = 1.73-84.52; $\mathrm{p}=0.012$ ). The conclusion of the study was that the Cebus population maintained in captivity in the northeast of Brazil is exposed to infection by antibodies anti-Leptospira and that corrective measures of animal handling, especially those related to daily hygienization of drinking fountains, must be implemented to reduce the risk of infection by serovars of Leptospira spp.
\end{abstract}

INDEX TERM: Leptospirosis, neotropical primate, epidemiology, Brazil.

\begin{abstract}
RESUMO.- Estudou-se a ocorrência de anticorpos anti-Leptospira e os fatores de risco associados à infecção em primatas do gênero Cebus mantidos em cativeiro no Nordeste do Brasil. Foram analisadas 139 amostras de soro sanguíneo de diferentes espécies de primatas de ambos os sexos e idades variadas. Para a pesquisa de anticorpos empregou-se a técnica de Soroaglutinação Microscópica (SAM) e para
\end{abstract}

\footnotetext{
${ }^{1}$ Recebido em 18 de agosto de 2011.

Aceito para publicação em 12 de setembro de 2011.

${ }^{2}$ Programa de Pós-Graduação em Ciência Veterinária, Departamento de Medicina Veterinária, Universidade Federal Rural de Pernambuco (UFRPE), Rua Dom Manoel de Medeiros s/n, Dois Irmãos, Recife, PE 52171900, Brasil. *Autor para correspondência: rinaldo.mota@hotmail.com

${ }^{3}$ Centro de Triagem de Animais Silvestres, IBAMA, BR $230 \mathrm{Km} \mathrm{10,} \mathrm{Cabe-}$ delo, PB 58310-000, Brasil.

${ }^{4}$ Unidade Acadêmica de Medicina Veterinária, Centro de Saúde e Tecnologia Rural (CSTR), Universidade Federal de Campina Grande (UFCG), Av. Universitária s/n, Bairro Santa Cecília, Cx. Postal 64, Patos, PB 58700-970, Brasil.
}

estudo dos fatores de risco utilizou-se análise multivariada. Foram observados anticorpos anti-Leptospira, sorovar Patoc em 6,5\% dos primatas (09/139). 0 fator de risco identificado nesse estudo foi a não higienização diária de bebedouros (Odds ratio=12,095; IC 95\% = 1,73-84,52; $\mathrm{p}=0,012$,). Conclui-se que a população de Cebus mantidos em cativeiros no Nordeste do Brasil está exposta à infecção por anticorpos anti-Leptospira e que medidas corretivas de manejo dos animais, especificamente aquelas relacionadas à higienização diária de bebedouros devem ser implementadas para reduzir o risco de infecção por sorovares de Leptospira spp.

TERMOS DE INDEXAÇÃO: Leptospirose, primata neotropical, epidemiologia, Brasil.

\section{INTRODUÇÃO}

Nas duas últimas décadas tem-se observado a emergência de algumas zoonoses associadas à interação de patógenos zoonóticos de animais selvagens, domésticos e humanos 
(Dszack et al. 2000) e a leptospirose apresenta-se como uma enfermidade de grande importância na Saúde Animal e Pública (OMS 2008).

No Brasil, a leptospirose é endêmica em áreas urbanas e rurais (Bharti et al. 2003), ressaltando-se a infecção por sorovares mantidos na natureza por animais silvestres (Aguiar et al. 2007). Em geral, as leptospiras podem infectar uma grande variedade de hospedeiros e existem sorovares que melhor se adaptam a cada hospedeiro (Kingscote 1986).

Esteves-Machado et al. (2010) relataram que fatores como predação, localização espacial arborizada, pontos de sombra, umidade alta, falta de higienização de bebedouros, circulação de animais sinantrópicos, entre outros, podem ser associados a maior predisposição à infecção por leptospiras em mamíferos, bem como o contato direto com a fonte de infecção.

A distribuição e ocorrência de anticorpos anti-Leptospira spp. nas populações de primatas neotropicais e diversos sorovares de Leptospira spp. já foram identificados em populações in situ e ex situ com destaque para os sorovares: Ballum, Icterohaemorhagie, Autumnalis, Pyrogenes, Panama, Pomona, Tarassovi, Canicola (Baulu et al. 1987), Castellonis, Copenhageni, Grippotyphosa (Corrêa et al. 2004), Pomona, Brasiliensis, Mini, Swajizak, Grippothyphosa, Sarmin, Fluminense, Autumnalis, Hebdomadis, Guaratuba, Javanica, Icterohaemorhagie (Souza Júnior et al. 2006), Cynopteri, Andamana, Hebdomadis, Copenhageni, Patoc, Cuíca, Hardjo, Icterohaemorrhagiae, Javanica, Grippotyphosa e Autumnalis (Costa 2009), Panama, Lai, Australis, Shermani e Patoc (Ibáñez-Contreras et al. 2010).

Esses achados mostraram que os primatas estão expostos a diversos sorovares de Leptospira spp. Informações sobre a ocorrência de infecção em primatas mantidos em cativeiro e os fatores de risco associados à infecção nessas condições ainda são escassas, considerando a diversidade de primatas existente no Brasil, sobretudo do gênero Cebus mantidos em cativeiro.

Desta forma, objetivou-se com este trabalho estudar a ocorrência de anticorpos anti-Leptospira spp. em Cebus spp. mantidos em Centros de Triagem de Animais Silvestres do Instituto Brasileiro do Meio Ambiente e Recursos Renováveis CETAS/IBAMA e zoológicos na região Nordeste do Brasil, bem como identificar os fatores de risco associados à infecção.

\section{MATERIAL E MÉTODOS}

A pesquisa foi autorizada pelo Instituto Chico Mendes de Conservação da Biodiversidade ICMBio/SISBIO sob número 16218$1 / 2008$ e comitê de ética da Universidade Federal de Campina Grande (UFCG), Protocolo 105/2009.

Foram coletadas amostras de sangue de 139 primatas do gênero Cebus de diferentes espécies, sexo e idades variadas, sendo cinco procedentes do CETAS/IBAMA de Alagoas (09 39' $57^{\prime \prime}$ Sul e $35^{\circ} 44^{\prime} 07^{\prime \prime}$ Oeste), 16 do CETAS/IBAMA de Pernambuco $\left(08^{\circ}\right.$ $03^{\prime} 14^{\prime \prime}$ Sul e $34^{\circ} 52^{\prime}$ 52" Oeste), 29 do CETAS/IBAMA da Paraíba (07 $06^{\prime} 54^{\prime \prime}$ Sul e $34^{\circ} 51^{\prime} 47^{\prime \prime}$ Oeste), 58 procedentes do CETAS/ IBAMA Rio Grande do Norte (05 $47^{\prime} 42^{\prime \prime}$ Sul e $35^{\circ} 12^{\prime}$ 34' Oeste), 19 do CETAS/IBAMA Piauí (05 05' 21" Sul e $42^{\circ} 48^{\prime} 07^{\prime \prime}$ Oeste), cinco animais do zoológico de João Pessoa/PB e sete do zoológico de Teresina/PI. As amostras foram coletadas no período de março de 2008 a fevereiro de 2010.

Para a coleta das amostras os animais foram contidos de forma física utilizando-se puçá e luvas de couro, seguida de contenção química utilizando-se associação de cloridrato de cetamina na dose de $10 \mathrm{mg} / \mathrm{kg}$, cloridrato de xilazina na dose de $1 \mathrm{mg} / \mathrm{kg}$ e diazepam na dose de $1 \mathrm{mg} / \mathrm{kg}$ administrados por via intramuscular.

Os animais foram submetidos ao exame clínico por meio da palpação, inspeção direta e indireta (Diniz 1997).

As amostras de sangue foram coletadas por venocentese da jugular, cefálica e/ou femoral, obtendo-se $5 \mathrm{~mL}$ de sangue com seringas e agulhas descartáveis.

Para cada animal foi preenchida uma ficha de identificação individual padrão do Centro de Proteção de Primatas Brasileiros contendo identificação do criadouro. Em cada estabelecimento foi aplicado um questionário com informações epidemiológicas sobre o manejo sanitário, nutricional, clínico e dados individuais dos animais.

Para a pesquisa de anticorpos anti-Leptospira spp.utilizou-se a técnica de Soroaglutinação Microscópica (SAM) de acordo com Galton et al. (1965) e Cole et al. (1973). Para a realização da SAM utilizou-se uma coleção de antígenos vivos procedentes do Laboratório de Doenças Transmissíveis da Universidade Federal de Campina Grande - Patos/PB que incluiu os seguintes sorovares: Australis, Bratislava, Autumnalis, Butembo, Castellonis, Bataviae, Canicola, Whitcombi, Cynopteri, Grippotyphosa, Hebdomadis, Copenhageni, Icterohaemorrhagiae, Javanica, Panamá, Pomona, Pyrogenes, Hardjo, Wolffi, Shermani, Tarassovi, Andamana, Patoc e Sentot. Os soros foram triados na diluição de 1:100 e aqueles que apresentaram $50 \%$ ou mais de aglutinação foram titulados por meio de diluições geométricas na razão dois. 0 título do soro foi a recíproca da maior diluição que apresentou resultado positivo. 0 ponto de corte utilizado foi o título 100 .

Para o estudo dos fatores de risco utilizou-se análise estatística univariada através do teste Qui-quadrado e as variáveis que apresentaram $\mathrm{p}<0,20$ foram selecionados para análise multivariada seguida de regressão logística. Valores do Odds Ratio (OR) e intervalo de confiança também foram calculados. 0 nível de significância utilizado nas decisões dos testes estatísticos foi de $5 \%$. Para a execução dos cálculos estatísticos utilizou-se o programa SPSS for Windows 12.0.

\section{RESULTADOS}

Das 139 amostras analisadas, nove $(6,5 \%)$ foram positivas para anticorpos anti-Leptospira sorovariedade Patoc com títulos que variaram de 100 a 400.

A frequência de anticorpos anti-Leptospira observada por Instituição foi de 40\% (02/05) no CETAS/IBAMA de Alagoas, $12,5 \%$ (02/16) no CETAS/IBAMA de Pernambuco e 8,6\% (05/58) no CETAS/IBAMA Rio Grande do Norte. Não foram registrados anticorpos anti-Leptospira spp. no CETAS/IBAMA da Paraíba, CETAS/IBAMA do Piauí, nos zoológicos de João Pessoa e Teresina.

$\mathrm{Na}$ análise univariada observou-se associação significativa $(p \leq 0,20)$ para os seguintes fatores: estado de procedência $(\mathrm{p}=0,05)$, fonte de água $(\mathrm{p}=0,006)$, não higienização diária de bebedouros $(\mathrm{p}=0,034)$ e tipo de bebedouro $(\mathrm{p}=0,013)$ (Quadro 1). Quando foram submetidos ao modelo final de regressão logística a única variável identificada como fator de risco foi a não higienização diária de bebedouros (OR=12,095 e p=0,012) (Quadro 2). 
Quadro 1. Frequência absoluta (n) e relativa (\%) da infecção por Leptospira spp. em Cebus spp. mantidos em CETAS e zoológicos de Alagoas, Pernambuco, Paraíba, Rio Grande do Norte e Piauí no período de 2008-2010

\begin{tabular}{|c|c|c|c|c|c|}
\hline \multirow[t]{3}{*}{ Variável } & \multicolumn{4}{|c|}{ SAM } & \multirow{3}{*}{$\begin{array}{l}\text { Valor } \\
\text { de } p\end{array}$} \\
\hline & \multicolumn{2}{|c|}{ Positivo } & \multicolumn{2}{|c|}{ Negativo } & \\
\hline & F.A. & F.R.(\%) & F.A. & F.R.(\%) & \\
\hline \multicolumn{6}{|l|}{ Fonte de água } \\
\hline Caixa de água e Companhia de água e esgotos & - & - & 33 & 100 & \multirow{4}{*}{$0,006^{*}$} \\
\hline Companhia de água e esgotos & 5 & 5,9 & 80 & 94,1 & \\
\hline Poço & 2 & 12,5 & 14 & 87,5 & \\
\hline Caixa e Poço & 2 & 40,0 & 3 & 60,0 & \\
\hline \multicolumn{6}{|l|}{ Higienização diária de bebedouro } \\
\hline Não & 2 & 40,0 & 3 & 94,8 & \multirow[b]{2}{*}{$0,034^{*}$} \\
\hline Sim & 7 & 5,2 & 127 & 60,0 & \\
\hline \multicolumn{6}{|l|}{ Estado de Procedência } \\
\hline Alagoas & 2 & 40,0 & 3 & 60,0 & \multirow{5}{*}{$0,05^{*}$} \\
\hline Paraíba & - & - & 34 & 100,0 & \\
\hline Pernambuco & 2 & 12,5 & 14 & 87,5 & \\
\hline Piauí & - & - & 26 & 100,0 & \\
\hline Rio Grande do Norte & 5 & 8,6 & 53 & 91,4 & \\
\hline \multicolumn{6}{|l|}{ Tipo de bebedouro } \\
\hline Vasilhame & 9 & 8,2 & 101 & 91,8 & \multirow[b]{2}{*}{0,113} \\
\hline Chupeta & - & - & 29 & 100 & \\
\hline \multicolumn{6}{|l|}{ Sexo } \\
\hline Macho & 4 & 4,9 & 77 & 95,1 & \multirow[b]{2}{*}{0,490} \\
\hline Fêmea & 5 & 8,6 & 53 & 91,4 & \\
\hline \multicolumn{6}{|l|}{ Tipo de criadouro } \\
\hline Cetas & 9 & 7,1 & 118 & 92,9 & \multirow[b]{2}{*}{1,000} \\
\hline Zoológico & - & - & 12 & 100,0 & \\
\hline \multicolumn{6}{|l|}{ Faixa etária } \\
\hline Filhote & - & - & 1 & 100,0 & \multirow{3}{*}{0,366} \\
\hline Juvenil & - & - & 23 & - & \\
\hline Adulto & 9 & 7,8 & 106 & 92,2 & \\
\hline \multicolumn{6}{|l|}{ Procedência dos Alimentos } \\
\hline Compra & 7 & 6,5 & 101 & 93,5 & \multirow[b]{2}{*}{1,000} \\
\hline Compra e doação & 2 & 6,5 & 29 & 93,5 & \\
\hline \multicolumn{6}{|l|}{ Armazenamento de ração } \\
\hline Balde & 7 & 9,1 & 70 & 90,9 & \multirow[b]{2}{*}{0,298} \\
\hline Balde e Saco & 2 & 3,2 & 60 & 96,8 & \\
\hline \multicolumn{6}{|l|}{ Armazenamento de frutas } \\
\hline Ambiente & - & - & 29 & 100 & \multirow[b]{2}{*}{0,204} \\
\hline Ambiente e geladeira & 9 & 8,2 & 101 & 91,8 & \\
\hline Higienização das frutas & & & & & \\
\hline Sim & 4 & 4,9 & 77 & 95,1 & 0.490 \\
\hline Não & 5 & 8,6 & 53 & 91,4 & 0,490 \\
\hline
\end{tabular}

Quadro 2. Análise multivariada dos fatores de risco para infecção por Leptospira spp. em Cebus spp. mantidos em CETAS e zoológicos de Alagoas, Pernambuco, Paraíba, Rio Grande do Norte e Piauí no período de 2008-2010

\begin{tabular}{lccc}
\hline Variável & Odds Ratio & I.C. 95\% & Valor de p \\
\hline $\begin{array}{l}\text { Não higienização } \\
\text { diária de bebedouros }\end{array}$ & 12,09 & {$[1,73-84,52]$} & 0,012
\end{tabular}

\section{DISCUSSÃO}

Os achados clínico-epidemiológicos sobre leptospirose em primatas ainda são escassos na literatura nacional e internacional. Neste estudo, os animais não apresentaram sinais clínicos sugestivos de leptospirose no momento da coleta das amostras, fato também observado por Pimentel et al. (2009) que relataram macacos-pregos saudáveis sem presença de sinais clínicos de leptospirose e também soropositivos para anticorpos anti-Leptospira.

Os resultados quanto à presença de anticorpos anti-Leptospira sorovar Patoc foram semelhantes àqueles descritos por Silva et al. (2010) que relataram esse sorovar em amostras de animais selvagens no estado de São Paulo, também estão de acordo com os achados obtidos por Costa (2009) que identificaram a infecção por esse sorovar em Cebus apella no Pará e Ito et al. (1998) em mamíferos silvestres do Pantanal matogrossense. Segundo a OMS (2008), o sorovar Patoc não causa enfermidade, porém, é possível encontrá-lo ocasionalmente em cultivos provenientes de material clínico.

A diversidade de sorovares de Leptospira spp. já descritos em estudos com primatas (Scarcelli et al. 2003 Corrêa et al. 2004, Esteves et al. 2005, Souza Junior et al. 2006, Andrade 2007, Pimentel et al. 2009) pode estar associada à presença dos reservatórios nos locais estudados. A infecção e transmissão de leptospiras estão relacionadas à exposição aos fatores de risco ambientais, incluindo o contato com animais silvestres ou domésticos (Escócio et al. 2010). Segundo Marinho (2008), do ponto de vista epidemiológico é importante o conhecimento das espécies animais que atuam como reservatórios, bem como a identificação dos sorovares prevalentes em determinada área. 
De acordo com Correa et al. (2004), Rattus novergicus é o animal sinantrópico responsável pela manutenção de leptospiras no ambiente do zoológico fato não mencionado nos questionários aplicados nesse estudo. Contudo, apesar desse reservatório não ter sido identificado nos recintos dos animais, acredita-se que estes possam circular, principalmente à noite e dessa forma contaminar a água ou alimentos.

Quando se avaliou a variável fonte de água, anticorpos anti-Leptospira foram observados em animais que recebiam água de três tipos diferentes de armazenamento: caixa de água e poço, poço e companhia de água e esgoto, reforçando a importância da água na cadeia epidemiológica da leptospirose. Provavelmente essa foi a fonte de infecção para os animais deste estudo já que a mesma apresentou associação significativa $(p=0,006)$ na análise univariada, embora não tenha despontado como fator de risco; resultado também relatado anteriormente por Costa (2009), que atribuíram à água a como a via de transmissão para primatas mantidos em cativeiro em Belém do Pará. Ainda de acordo com Picardeu et al. (2008), Leptospira biflexa é uma espiroqueta saprófita de vida livre presente em meio aquático.

A não higienização diária de bebedouros foi o fator de risco identificado neste estudo. Os animais procedentes de instituições que não realizavam a higienização diária dos bebedouros apresentaram 12,09 mais chances de infecção para o sorovar Patoc. Esse achado reforça o que já se conhece sobre a epidemiologia da leptospirose nos animais domésticos e esse aspecto do manejo deve ser observado com cuidado pelos tratadores de primatas. A higienização periódica dos reservatórios de água, de bebedouros e a troca de bebedouros tipo canaleta por bebedouros automáticos são medidas preventivas recomendadas no controle da leptospirose (Delbem et al. 2004).

Pimentel et al. (2009) enfatizaram ainda que a prevenção da leptospirose em cativeiro deve apoiar-se em mudanças de hábitos no manejo sanitário, na capacitação de pessoal, na vigilância, bem como no monitoramento sorológico rotineiro nos animais selvagens. Ainda de acordo com Ito et al. (1998) para animais silvestres, os estudos soroepidemiológicos constituem-se em uma importante ferramenta para monitorar as doenças de interesse em saúde animal e saúde pública.

\section{CONCLUSÕES}

A população de Cebus, mantida em CETAS e Zoológicos na região nordeste do Brasil, está exposta à infecção por Leptospira, sorovar Patoc.

De acordo com o fator de risco identificado nesse estudo, medidas corretivas de manejo dos animais mantidos em cativeiro, especificamente relacionadas à higienização diária de bebedouros devem ser implementadas para reduzir o risco de infecção por esse e outros sorovares de Leptospira spp.

Agradecimentos.- À CAPES pela concessão da bolsa de estudos concedida à Débora R.A. Ferreira, aos CETAS/IBAMA e Zoológicos do Nordeste do Brasil, por permitirem a coleta do material biológico, e aos seus técnicos, tratadores e estagiários por toda colaboração.

\section{REFERÊNCIAS}

Aguiar D.M., Cavalcante G.T. \& Marvulo M.F.V. 2007. Fatores de risco associados à ocorrência de anticorpos anti Leptospira spp. em cães do município de Monte Negro, Rondônia, Amazônia Ocidental Brasileira. Arq. Bras. Med. Vet. Zootec. 59:70-76.

Andrade T.M. 2007. Títulos de anticorpos contra Leptospira spp. e análise bioquímica no soro sanguíneo em macaco prego (Cebus apella nigritus). Tese de Doutorado em Medicina Veterinária, Universidade Estadual Paulista Julio de Mesquita, Jaboticabal. 62p.

Baulu J., Everard C.O.R. \& Everard J.D. 1987. Leptospires in vervet monkeys (Cercopithecus aethiops Sabaeus) on Barbados. J. Wildl. Dis. 23:60-66.

Bharti A.R., Nally J.E., Ricaldi J.N., Matthias M.A., Diaz M.M., Lovett M.A., Levett P.N., Gilman R.H., Willig M.R., Gotuzzo E. \& Vinetz J.M. 2003. Leptospirosis: a zoonotic disease of global importance. Lancet Infect. Dis. 3:757-771.

Cole Junior J.R., Sulzer C.R. \& Pursell A.R. 1973. Improved microtechnique for agglutination test. Appl. Microbiol. 25:970-980.

Corrêa S.H.R., Vasconcellos S.A., Morais Z., Teixeira A.A., Dias R.A., Guimarães M.A.B.V., Ferreira F. \& Ferreira Neto J.S. 2004. Epidemiologia da leptospirose em animais silvestres na Fundação Parque Zoológico de São Paulo. Braz. J. Vet. Res. Anim. Sci. 41:189-193.

Costa S.M. 2009. Estudos da frequência de anticorpos contra Leptospira interrogans e Trypanossoma cruzi em soros sanguíneos de primatas neotropicais de cativeiro. Dissertação de Mestrado em Ciência Animal, Universidade Federal do Pará, Pará. 91p.

Daszack P., Cunningham A.A. \& Hyatt A.D. 2000. Emerging infectious diseases of wildlife: threats to biodiversity and and human health. Science 287:443-449.

Delbem A.C.B., Freire R.L., Silva C.A., Müller E.E., Dias R.A., Ferreira Neto J.S. $\&$ Freitas J.C. 2004. Fatores de risco associados à soropositividade para leptospirose em matrizes suínas. Ciência Rural 34:847-852.

Diniz L.S.M. 1997. Primatas em cativeiro: manejo e problemas veterinários. Ícone, São Paulo. 197p.

Epiphanio S., Catão-Dias J.L. \& Guimarães M.A.B.V. 1999. Toxoplasmosis in emperor tamarin (Saguinus imperator): case report. Braz. J. Vet. Res. Anim. Sci. 36:72-74.

Escócio C., Genovez M.E., Castro V., Piatti R.M., Gabriel F.H.L., Chiebao D.P., Azevedo S.S., Vieira S.R. \& Chiba M. 2010. Influência das Condições Ambientais na Transmissão da Leptospirose entre Criações de Ovinos e Bovinos da Região de Sorocaba, SP. Arqs Inst. Biológico, São Paulo, 77:371-379.

Esteves F.M., Guerra-Neto G., Girio R.J.S., Silva-Vergara M.L. \& Carvalho A.C.F.B. 2005. Detecção de anticorpos para Leptospira spp. em animais e funcionários do zoológico municipal de Uberaba, MG. Arqs Inst. Biológico, SãoPaulo, 72:283-288.

Esteves-Machado F.M., Coelho H.E. \& Rezende R.S. 2010. Plano de ação para o controle da Leptospirose no zoológico Municipal de Uberaba/ MG. Biosci. J. 26:981-989.

Galton M.M., Sulzer C.R., Santa Rosa C.A. \& Fields M.J. 1965. Aplication of a microtecnique to the aglutination test for leptospiral antibodies. Appl. Microbiol. 13:81-85.

Garcia J.L., Svobodo W.K., Chryssafidis A.L., Malanski L.S., Shiozawa M.M., Aguiar L.M., Teixeira G.M., Ludwig G., Silva L., Hilst C. \& Navarro I.T. 2005. Sero-epidemiological survey for toxoplasmosis in wild New World monkeys (Cebus spp., Alouatta caraya) at the Paraná river basin, Paraná State, Brazil. Vet. Parasitol. 133:307-311.

Ibáñez-Contreras A., Hernández-Godínez B., Torres-Barranca J.I. \& Meléndez-Vélez P. 2010. Hallazgos de anticuerpos contra Leptospira sp., serovariedades Panama, Lai, Australis, Shermani y Patoc, en un grupo de monos rhesus (Macaca mulatta) en condiciones de cautiverio. Arch. Med. Vet. 42:101-104.

Ito F.H., Vasconcellos S.A., Bernardi F., Nascimento A.A., Labruna M.A. \& Arantes I.G. 1998. Evidência sorológica de brucelose e leptospirose e parasitismo por ixodídeos em animais silvestres do pantanal mato-grossense. Ars Vet. 14:302-310. 
Kingscote B.F. 1986. Leptospirosis outbreak in piggery in Southern Alberta. Can. Vet. J. 27:188-190.

Marinho M. 2008. Leptospirose: fatores epidemiológicos, fisiopatológicos e imunopatogênicos. Vet. Zootec. 15:428-434.

OMS 2008. Leptospirosis humana: guia para el diagnóstico, vigilância y control. Organización Mundial de la Salud, Rio de Janeiro. 127p.

Picardeau M., Bulach D.M., Bouchier C., Zuerner R.L., Zidane N., Wilson P.J., Creno S., Kuczek E.S., Bommezzadri S., Davis J.C., McGrath A., Johnson M.J., Boursaux-Eude C., Seemann T., Rouy Z., Coppel R.L., Rood J.I., Lajus A., Davies J.K., Médigue C. \& Adler B. 2008. Genome sequence of the saprophyte Leptospira biflexa into the evolution of Leptospira and pathogenesis of leptospirosis. Plus One 3:1-9.

Pimentel J.S., Gennari S.M., Dubey J.P., Marvulo M.F.V., Vasconcellos S.A., Morais Z.M., Silva J.C.R. \& Evêncio Neto J. 2009. Inquérito sorológico para toxoplasmose e leptospirose em mamíferos selvagens neotropicais do Zoológico de Aracaju, Sergipe. Pesq. Vet. Bras. 29:1009-1014.
Scarcelli E., Piatti R.M., Fedullo D.L., Simon F., Cardoso M.V., Castro V., Miyashiro S. \& Genovez M.E. 2003. Leptospira spp. detection by Polymerase Chain Reaction (PCR) in clinical samples of capitive Blackcapped capuchin monkey (Cebus apella). Braz. J. Microbiol. 34:43-146.

Silva F.J., Mathias L.A., Magajevski F.S., Werther K., Assis N.A. \& Girio R.J.S. 2010. Anticorpos contra Leptospira spp. em animais domésticos e silvestres presentes no campus universitário da FCAV, Unesp, Jaboticabal/ SP. Ars Vet; 22:17-25.

Silva J.C.R. 2005. Zoonoses e doenças emergentes transmitidas por animais silvestres. Disponível em <www.abravas.org.br> Acesso em 16 jul. 2005.

Souza Júnior M.F., Lobato Z.I.P., Lobato F.C.F., Moreira E.C., Oliveira R.R., Leite G.G., Freitas T.D. \& Assis R.A. 2006. Presença de anticorpos da classe IgM de Leptospira interrogans em animais silvestres do estado do Tocantins, 2002. Revta Soc. Bras. Med. Trop. 39:292-294.

Szonyi B., Agudelo-Flórez P., Ramírez M., Moreno N. \& Ko A.I. 2010. An outbreak of severe leptospirosis in capuchin (Cebus) monkeys. Vet. Journal, doi: 10.1016/j.tvjl.2010.05.02. 